\title{
ON THE THEORETICAL EXAMINATION OF THE ADOPTION OF FORMAL METHODS IN THE RAILWAY SIGNALING SECTOR
}

\author{
Dimitrios Rizopoulos ${ }^{1}$, Nils O.E. Olsson ${ }^{2}$, Anders Lindahl ${ }^{3}$, Olov Lindfeldt ${ }^{4}$ \\ 1,3 KTH Royal Institute of Technology, SE-100 44 Stockholm, Sweden \\ ${ }^{2}$ NTNU Norwegian University of Science and Technology, NO-7491 Trondheim, Norway \\ ${ }^{4}$ MTR Pendeltågen, Rålambsvägen 17, SE-112 59 Stockholm, Sweden
}

Received 12 April 2021; accepted 28 June 2021

\begin{abstract}
It is now more evident than ever before that the organizations that develop or utilize railway signaling systems need to take advantage of modern scientific disciplines and technologies in order to meet transportation demand, improve train services, and re-assure the financial and environmental sustainability of railways. Although several game-changing technologies have emerged both in academic studies and the industry, adoption has differed across industries and sectors, with some of them employing modern tools and extracting their benefits, while others not. While this phenomenon can be attributed to the levels of demand for technological solutions according to the needs of each market, on the other hand, it can be accredited to the unsuccessful attempt to understand how the implementation of adoption itself could take place. In the current article, it is discussed how the study of the adoption of Formal Methods, and the tools that can be developed based on them, can occur in a systematic way in order to extract critical insights for this process. The analysis included in this article is part of the on-going discussion on the systematic study of the adoption of emerging technologies in railways and the currently developed scientific literature on the topic.
\end{abstract}

Keywords: adoption, railways, signaling, formal methods.

\section{Introduction}

Over the last few decades, European railway systems are changing their organization from state-owned monopolies to deregulated and restructured systems, usually with a separation between ownership and operation of the infrastructure. Railway investment projects across several types of engineering disciplines (civil and construction work, signaling equipment, electricity supply, telecommunications, railway tracks) are affected by those changes, as well as the procurement strategies that IMs follow in order to acquire solutions that will futureproof their services.

Across the many structural changes that are coming to the railways and the signaling industry due to emerging technological and societal needs (Smith, 2003; Batty et al., 2012), one of the main challenges is to successfully adopt emerging technologies and innovate in terms of practices followed and overall efficiency (Iacono et al., 2012). One of the scientific fields, and associated technologies, that has the potential to facilitate a lot of positive changes in the

\footnotetext{
${ }^{2}$ Corresponding author: nils.olsson@ntnu.no
} 
sector is the field of Formal Methods (FMs). This article studies the utilization of FMs, and tools that are based on them, from the perspective of their adoption as an innovation in railway signaling.

Various meanings have been considered for the term innovation. While in some studies, innovation has been thought of as a process (Baregheh, Rowley and Sambrook, 2009) from which ideas conceived are brought to reality, in other articles, it can have a more of a market-based meaning (Kline, 1985), (Hauschildt et al., 2016), such as a solution that satisfies business needs in new ways. Edison et al. (2013) analyzed several definitions for coverage against aspects of the meaning of innovation, as identified across studies in the scientific literature. The most suitable was decided to be the one from Crossan and Apaydin (2010), who in their article state that: "Innovation is: production or adoption, assimilation, and exploitation of a value-added novelty in economic and social spheres; renewal and enlargement of products, services, and markets; development of new methods of production; and establishment of new management systems. It is both a process and an outcome". While innovation, and especially technological one, has been proven to have an impact (Coccia, 2009) on society and organizations, it is not always the case its adoption is successful (Douthwaite, Keatinge and Park, 2001; Ren, 2019).

Regarding FMs themselves, the term includes all the mathematical based methods that allow the rigorous specification of cyberphysical systems, their formal development, and verification through mathematical proof. Several use cases and benefits, as they are described in later sections of this article, stem from the application of
FMs, with the main one being the formal development of systems, which in turn leads to a guaranteed safety level and compliance with international standards. Except for formal development, one could claim that a significant challenge in the adoption of FMs in railway signaling is to present other use cases that can improve railway signaling systems and their architecture, interoperability, and integration, and hopefully, in the long run, lower Life Cycle Costs (LCC) and improved efficiency in signaling projects. It can be considered that the improvements in the signaling systems themselves can also affect the train services in the long term, with services' punctuality (Palmqvist, Olsson and Hiselius, 2017) or track capacity (Goverde, Corman and D'Ariano, 2013) being directly connected to signaling systems in previous studies.

In order to extract the benefits of the utilization of formal methods tools, their adoption needs to be implemented. In this process, stakeholders in railway signaling projects, on the one hand, have to get familiar with the possibilities and results of FMs use and, on the other hand, have to acquire the procedural knowledge (i.e., know-how) of how to apply FMs on their future projects and applications. Successful adoption of an innovation can also be considered as the case when an organization, and its members at all levels, can utilize a technology or a tool when required. The field of FMs application to railway signaling is only now starting to accumulate and formalize the knowledge that has been acquired through research in both the academic and the industrial settings regarding possible applications (Rizopoulos et al., 2020). Although there are several mature studies (Haxthausen, 2010; Gruner et al., 2013; Fantechi, Flammini and Gnesi, 2014; Gruner, Kumar and Maibaum, 2016) 
on a higher-level that review FMs tools and applications themselves, there are only a few reports (Bacherini et al., 2006; Ferrari, Fantechi and Gnesi, 2012; Ferrari et al., 2013; Huhn and Milius, 2014; Basile et al., 2018; ter Beek et al., 2019) address the adoption of FMs, or include related references to adoption, its implementation, and the possible research questions.

Studying, explaining, and forecasting the adoption of technologies is a very crucial step towards reaching the full potential of the utilization of one scientific discipline and related technologies. Usually, implementing adoption is related to investment in the innovation and research programs that can facilitate its better understanding within an organization. Those same investments can fail and can lead to financial loss but also dissatisfaction for employees (Venkatesh, 2000). Thus, identifying determinants of successful adoption is not a task to be neglected, but rather a crucial step towards innovation.

The purpose of this article is to lay a solid foundation in the discussion around the systematic study of FMs adoption in railways and signaling. The authors believe alongside the efforts that go into developing new tools based on game-changing scientific disciplines such as FMs, the study of how these tools can be adopted should be prioritized so that investments can be productive and successful. Furthermore, the analysis that is presented in this article is based on qualitative research and first-hand observations of trends in modern signaling projects. For the bibliographic research that has been included, articles and reports have been sourced from online databases and platforms (ScienceDirect, Google Scholar, ResearchGate, and Web of Science).
In that regard, Section 2 gives an overview of how FMs are currently utilized in the signaling sector, what are potential use cases and what is the current state of adoption. In Section 3, we provide some ways in which a systematic study of adoption can be conducted in the future. In the conclusion section, overall remarks and research directions are discussed.

\section{Formal Methods in Railway Signaling}

\subsection{Description}

Formal Methods (FMs) are one of those scientific fields that can improve cyberphysical systems through tools that can be used for the rigorous analysis of the behavior of software and hardware of the systems in scope. They have been extensively utilized in several industries that employ safety, security, and business-critical systems. In the case of railway signaling, they can be used in order to develop the signaling apparatus according to international standards but can also be included in other use cases outside the formal development of systems. Alongside FMs for the development of systems, semiFormal Methods (semi-FMs) can be used, which are not guaranteed to maintain complete mathematical formality but can still provide benefits to users, similar to those that FMs can offer. While the terms FMs and semi-FMs are usually used to refer to a field of applied science, in this article, we interchangeably use these terms to refer to also refer to all the technological tools that fall under the umbrella of FMs and semiFMs.

It can be considered that the demand and essential use cases of FMs stem from legislation and international guidelines for the development of signaling apparatus. 
The most prominent example within signaling is EN50128 guidelines by the European Committee for Electrotechnical Standardization (CENELEC), which "specifies the process and technical requirements for the development of software for programmable electronic systems for use in railway control and protection applications". However, in this article, several use cases are presented and analyzed that do not stem from legislation or solely concern development, but also other functions that need to take place in the attempt of railway signaling organizations to develop modern systems. Such processes and functions could, for example, be the procurement process or the validation of safety that sometimes need to take place outside the development (not by the supplier of the software system but from an IM or an assessor).

\subsection{Use Cases and Benefits}

Several use cases have been presented and discussed in the scientific literature that can benefit organizations in railway signaling. The first use case can be considered to be the formal development of systems, where FMs are utilized to study and validate the behavior of software and hardware systems. This use case can be considered to be the most widely demonstrated across the literature, among other use cases. Examples exist based on the type of system, such as the article by Essamé et al. (2006), which presents the application of the B method for safety-critical software of the New York City Canarsie Line. Another example is the report by Behm et al. (1999), who presented the processes followed for the development of an automatic train operating system for the Paris Metro line 14 alongside insights about the organization and team's roles within the project. Finally, successful instances of FMs applications exist, from both the academic (Haxthausen, 2010; Vu, Haxthausen and Peleska, 2014; Vu, 2015) and the industrial perspective (Cimatti et al., 1998), for interlocking systems which are assigned with the highest Safety Integrity Level 4 (SIL4) in CENELEC's EN50128 guidelines.

Moving on, another interesting use case for FMs is their utilization in developing reference models for a safety-critical industry, thus enabling the reference model-based development of systems by suppliers in that industry. As a first step towards this use-case, the reference model can be developed by a group of experts in the attempt to portray a comprehensive architecture for a type of system. This use case is especially useful since the reference model will developed according to international standards and will have formally proven behavior. In addition, the reference model can include several levels of granularity: for example, it can possibly include the subsystems and the relations between them in the architecture model, or it can possibly include a specific toolchain to be used for developing an instance of the system in scope.

A reference model can be utilized across several projects and organizations for effective communication on a common reference basis. Benefits from utilizing such a use case can range from the re-usability of artifacts and increased productivity to improved communication between stakeholders in a project. Related discussion on this use-case is presented in the European research project called openECTS (Karg et al., 2016), which aims at formalizing the system requirements specification of the European Train Control System (ETCS) and a reference implementation of the system based on an open-source toolchain. A depiction of this use case is provided in Figure 1 below. 


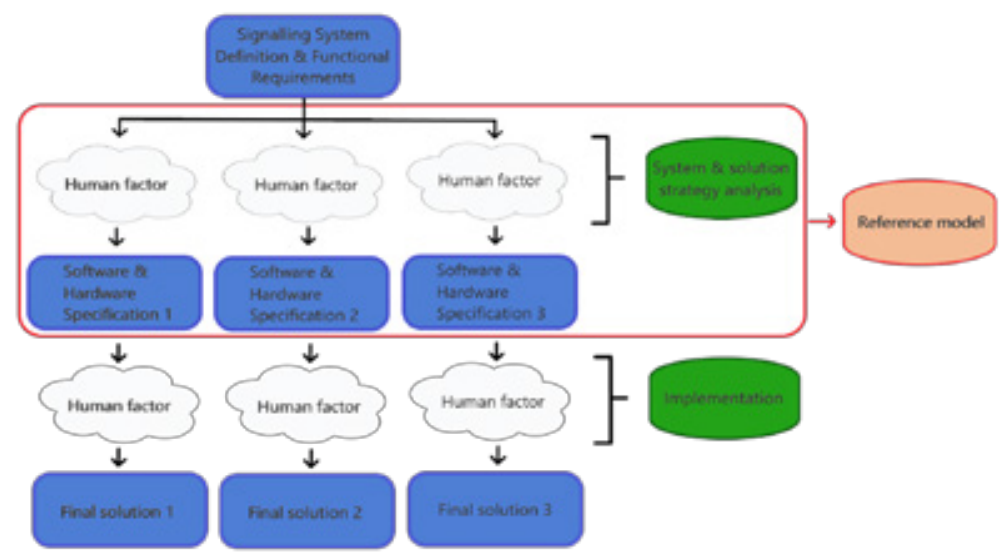

Fig. 1.

An Illustration of a Generic Railway Signaling Solution Development Process and how the Encapsulation of a Part of that Process can be Included in a Reference Model

Lastly, the procurement process itself can benefit from the applications of FMs. FMs can be used for the tender-based safety verification of requirements for systems as they are set by IMs. In this use case for FMs, the IM specifies the safety requirements for the signaling solution that is part of the tender scope. The safety requirements are more elaborated and in a rigorous form (this can be realized with semi-FMs as well), as the requirements are intended to be verified, using formal verification against formal models of the system developed by several suppliers. In that regard, this use case results in a more reliable process of the verification of safety for a system (which can be developed by one or multiple suppliers) against a "standard" set of safety requirements formulated by the IM. This use case can result in several benefits, with the main one being the reduced effort towards the safety verification and compliance validation with CENELEC EN50128 of systems delivered, when several suppliers deliver the same type of solution in a procurement process.

As a summary, some of the benefits that formal methods enable are:

- The automated development of verified signaling systems and subsystems that comply with international standards;

- The validation of systems for the expected behavior (a topic that is especially important in safety, security, and business-critical industries);

- Through the application of FMs or semiFMs in the early stages of a project life cycle: We can have the improvement of the specification, design, and architecture of a system;

- The re-usability of artifacts from a single product development process into multiple projects;

- The improvement of accuracy in the specification of requirements tasks in procurement processes; 
- In the long term, and through widespread adoption in industry, cost reduction and time savings of projects' through the application of FMs or semiFMs on multiple levels of a project.

\subsection{Concerns Regarding the Adoption of FMs}

The study of the adoption of FMs tools has been initialized in several other reports (Bacherini et al., 2006; Ferrari, Fantechi and Gnesi, 2012; Ferrari et al., 2013; Huhn and Milius, 2014; Basile et al., 2018; ter Beek et al., 2019). Despite the potential benefits, the adoption of FMs in railway signaling can be considered not to be complete yet as organizations hesitate to invest or utilize FMs tools in their projects. From all of the available material that discusses adoption, it can be noticed that some potential crucial factors such as the definition of the learning curve (considering ease of use, documentation, and available support) for toolkits and compatibility across tools of toolkits can be substantial. While there is not, however, a systematic longitudinal study or approach to the topic of adoption, several research questions arise (Rizopoulos et al., 2020), and strategical approaches on how to integrate FMs into current processes and projects will be hard to be developed.

Although it can be hard to compare without a quantitative assessment, one could claim that this is not the case for other industries though, where adoption can be considered to have progressed in a faster and more effective way than the case of railway signaling. Several examples and comprehensive studies (Woodcock et al., 2009) exist within industries, such as the automotive industry, medical devices, computer software, and hardware systems. For instance, let us refer to the avionics industry, where many more studies are available, both from the industrial and academic settings. Maybe the most prominent examples of reviews are the ones conducted by Souyris et al. (2009) and Laurent (2010), where the integration of FMs tools and verification techniques into the development of avionics software products of Airbus aerospace company. This can be attributed to the fact that the railways' industry and the signaling sector can be considered to be a conservative industry, and an implementation lag can be found. On a higher level, this lag in the adoption can be partially attributed to the general misconceptions that regard FMs regardless of the application sector. Those generic misconceptions, that experienced individuals in the field of FMs are most likely already aware of, have been available in the scientific literature since 1990 in reviews (Hall, 1990; Bowen and Hinchey, 1995). Overall, they concern misconceptions regarding the easeof-use, the benefits of use, or even concerns regarding the support that FMs tools usually get from their developers.

Regarding some concerns that can potentially exist with FMs applications in railway signaling:

- Although FMs, semi-FMs, and tools that are based on them enable the mathematical analysis of the behavior of systems, still this analysis is subject to the perspective of the modeler or practitioner using the tool. This means that reducing the effect that the human factor can have on the analysis is possible, but it is still hard to fully eliminate it, still theoretically allowing (although it is not common) software errors to show up if the practitioner is not qualified enough or does not have the right expertise; 
- One could claim that FMs and related tools have been available for a few decades now, although it seems like there has never been enough market pressure to create significant demand for use cases outside legislation;

- Regarding reference models that can be established in signaling: The consensus and collaborative effort required for such a step can be underestimated;

- By using FMs and specific tools for applications or initiatives like reference models, demand for certain tools can rise, but the demand for other tools may reduce, leading to more vendor lock-in regarding the FMs tools themselves.

\section{On the Systematic Study of Technology Adoption in Railway Signaling}

\subsection{Review of the Topic of Technology Adoption Theories and Frameworks}

Important insights can be gathered for the understanding of the adoption of an innovative technology by applying an adoption theory or framework. More specifically, a technology acceptance model overall attempts to explain user intentions to use an information system and the expected user behavior, usually through several stages of adoption. While several types of technologies have been studied, a big part of the modern literature focuses on emerging information systems, which can be defined as "a formal, sociotechnical, organizational system designed to collect, process, store, and distribute information. In a sociotechnical perspective, information systems are composed of four components: task, people, structure (or roles), and technology" (O'Hara, Watson and Kavan, 1999). In that light, the aim of the proposed research in this sub-section is to study the applications of FMs, and the tools or toolkits that can potentially be utilized for the use cases by organizations that are stakeholders in signaling projects.

Several works exist in the field of adoption of innovations, whether innovation refers to technological advancements or other beneficial sets of changes for organizations. To begin with, in the book Diffusion of Innovations (DoI) (Rogers, 2003), which has become one of the most pivotal textbooks on diffusion studies and the spreading of trends that regard innovation. The qualities that play a role in this dissemination of news ways of covering needs are given, and the importance of communication is highlighted within networks of peers. Fundamental is the classification of users into groups with different characteristics, who are considered to react differently to innovation and its effects.

Other well-established theories that exist concern the diffusion of new technologies and innovations. One approach is the Concerns-Based Adoption Model (CBAM) that has been initially conceptualized by Frances Fuller (1969) and later developed by Gene E. Hall (1979). CBAM is a model based on the perspective of individuals that are subjected to adapt and change and concerns the educational and academic aspects of the facilitation of this change. Based on the experience of the authors in innovation adoption in the education sector and the industry, several stages of concern are introduced (Hall, 1979) for teachers, or, broadly speaking, experienced change agents in their field, where innovation adoption needs to occur. A significant advantage of CBAM is that it can be applied to real-world situations and case studies, in contrast to the Diffusion of Innovations theory. Such 
a case study is the work by Christou et al. (2004), where the concerns of teachers are studied in connection to the introduction of an innovative mathematics curriculum in primary schools of Cyprus. The results showed that teachers accepted the changes and did not seem to concern themselves regarding specific innovation tasks.

While DoI and CBAM are concerned with the spread of new ideas in any field, some theories have been developed specifically with respect to the adoption of technology and information systems. One of the most discussed and studied theories is the Technology Acceptance Model (TAM) theory, which is based upon the Theory of Reasoned Action, as discussed by Ajzen (1985). TAM has been initially presented by Davis (1989) and is based around two variables that define technological adoption, which are the potential users' perceived usefulness and perceived ease-of-use of the technology. Davis also provides valid measures for the two variables that stem from two studies that involve a sample of 152 individuals. An interesting study that applies TAM for the better understanding of an e-ticket booking service for the Indian railways has been presented by Puthur et al. (2020).

TAM was extended to the Technology Acceptance Model 2 (TAM2) in 2000 by Venkatesh et al. in two main contributions (Venkatesh and Davis, 2000), (Venkatesh, 2000), where, in essence, TAM is extended to include the effect of social influence and cognitive instrumental processes. One of the significant contributions that are stated in former of the two referenced articles (Venkatesh and Davis, 2000), that extends TAM regarding the variable of perceived usefulness, the findings show that the demonstration of the effectiveness of the new tool as compared to the status quo technology may lead to increased acceptance by the users. A later article extends the understanding for the second parameter, the perceived ease of use, and the determinants that affect it. One of the main contributions stated in the article is that although the user interface is an essential factor that determines the perceived easeof-use, other factors play an indispensable role that is not directly connected to the user-system interaction. They also found that computer self-efficacy and the individual's opinions regarding computers are the most critical determinants of perceived easeof-use. Finally, Technology Acceptance Model 3 (TAM3) was introduced in 2008 by Venkatesh et al. (2008). In contrast to previous approaches that address technology adoption from the user-perspective, in TAM3, an organizational view is also given alongside the user/employee view. Concerning the organizational aspect of the adoption process, TAM3 also serves as a framework for managerial decisions that can be introduced prior to or after the implementation of the innovation process.

A final vital framework that a reference will be made to is the work by Venkatesh et al. (2003), where the authors reviewed eight prominent models for technology adoption and introduced a new unified model called Universal Technology Adoption and Use Theory (UTAUT) model, and validate it through surveys. By capturing the most practical and essential aspects of 8 models in UTAUT, four key constructs, and the relationship between them, are considered as the most critical determinants for user intentions to utilize a new information system and their corresponding usage behavior. Namely, they are $i$ ) performance 
expectancy ii) effort expectancy iii) social influence, and $i v$ ) facilitating conditions, while the research model also accounts for the role of key moderators such as gender, age, voluntariness, and experience. UTAUT, in the empirical validation by longitudinal field studies in four organizations, outperforms all the other eight models considered and includes a sophisticated multi-level approach while emphasizing the contextual analysis of adoption. Below, in Figure 1, the research model for UTAUT is provided, as introduced by Venkatesh et al. (2003).

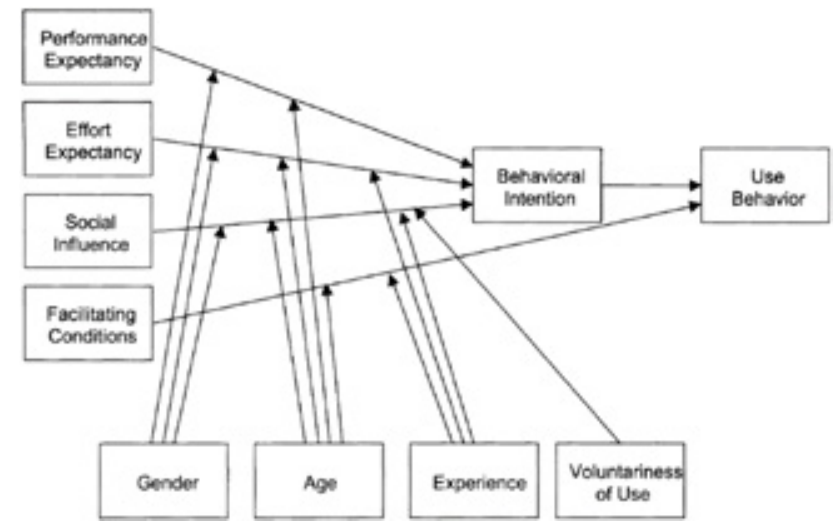

Fig. 2.

A Depiction of Variables in the UTAUT Research Model

Source: Venkatesh et al. (2003)

Extensions of the initial UTAUT model exist, such as the UTAUT2 model (Venkatesh, Thong and $\mathrm{Xu}, 2012$ ). Regarding the application of such a framework, in the case of the initial UTAUT model, one could claim that it is the most prominent framework used in modern literature with the original paper being cited 27529 times (at the time of writing this article), several examples can be taken from previous studies that study technologies that range from course management software (Marchewka, Liu and Kostiwa, 2007) to internet banking (Foon and Fah, 2011).

Interesting applications that indicate that UTAUT can be used for studying the adoption of FMs tools in railway signaling can be found in the literature. Related to the railways sector is the article by Indrawati $e t$ al. (2017), where the authors present how the study of adoption factors for an online ticket reservation system is conducted. Furthermore, another study is the one by Madigan et al. (2016), which falls outside the umbrella of information systems, but within the transportation domain and showcases how UTAUT can be adapted and utilized to investigate the factors which might influence acceptance of automated road transport systems. Overall positive insights are shared in this initial publication since the application of UTAUT was able to bring up some insights about adoption for the systems studied, with the authors highlighting that further adaptation of the framework to include other factors or concepts could further increase the understanding of 
adoption. Such an adaptation of UTAUT in the case of studying the adoption of automated road transport vehicles would be the inclusion of hedonistic motivation (users' enjoyment of using the system) in driving. In a later article by Madigan et al. (2017), which can be considered as the continuation of the initial study (Madigan et al., 2016), the social-psychological model is utilized to study the intentions of users of automated road transport systems during their actual demonstration in the city of Trikala, Greece. The modified UTAUT model considered in the study successfully helps researchers understand more things about how users perceive the innovative technology to be used. Finally, another application of UTAUT within the transportation science domain is the study by Ye et al. (2020), who study the concept of Mobility as a Service (MaaS) and its adoption, and how the potential users of such services, in a small town called Anting New Town in the suburbs of Shangai, perceive them. With the application of UTAUT, the authors were able to identify that convenience was the most substantial demand, with more impact on acceptance rather than time and cost. One could claim that the derivation of such an insight would be really hard without the application of such a framework and without a systematic approach to adoption. Based on these types of observations and corresponding analysis, the authors (Ye, Zheng and Yi, 2020) were able to come up with suggestions and strategies for the improved promotion of $\mathrm{MaaS}$ in the region, and consequently, more successful adoption.

All of the aforementioned theories, models, and frameworks are proven approaches that can be extremely useful with respect to our topic. The scope of this section is not to compare adoption theories, which can be found across several works in the literature (Venkatesh et al., 2003; Straub, 2009), but rather to provide an initial research direction towards studying FMs adoption in railway signaling. However, the need for the selection of one of those theoretical frameworks should be highlighted and is part of future research work. The theory of choice should have the following characteristics:

- Broad foundation and acceptance;

- Cross-field approach to diffusion adoption that ranges across the fields of education, sociology, psychology, and others;

- It should be proven to provide useful insights into the adoption of innovation in other areas of technology and industry.

UTAUT model satisfies all aforementioned conditions, but other frameworks may be used in such a study. By applying the innovation of adoption theory to the case of FMs in signaling systems in railways, it is expected that a solid understanding of the structure of adoption will be gained in this direction of research. While the railways' sector is considered to be a conservative one, getting insights on which determinants will allow individuals to be more open to using new technologies can be beneficial not only in the case of FMs but in other cases of technology adoption in railways.

\subsection{Next Steps in the Application of UTAUT to Study the Adoption of FMs in Railway Signaling}

Possibly, an extension to the UTAUT framework may be required in order to study the adoption of FMs. This would happen through several determinants that may be considered important for the field of FMs in railway signaling but not for other 
information systems, as they were studied and presented in previous studies described in Section 3.1 of this article.

Such determinants can be sourced from the literature that already exists on the adoption of FMs. From the article by Rizopoulos et al. (2020), where a review of articles that concern the state of adoption in railway signaling is presented, several factors for FMs adoption can be extracted. As included in (Rizopoulos et al., 2020), some of the most important articles (Bacherini et al., 2006; Ferrari, Fantechi and Gnesi, 2012; Ferrari et al., 2013; Huhn and Milius, 2014; Basile et al., 2018; ter Beek et al., 2019) on the adoption of FMs in railway signaling indicates that potential crucial factors for adoption are:

- Adoption should be studied from both an academic and industrial setting;

- Definition of the learning curve (considering ease of use, documentation, and available support) for toolkits can be beneficial, especially from an individual user perspective;

- Compatibility across tools of toolkits can be substantial;

- On a general level, formal specification, formal verification, and formal modeling are the most relevant features, as observed from questionnaire-based studies.

Additionally it can be noticed that also the factors that play a role in the adoption of FMs, as identified by previous studies (Bacherini et al., 2006; Ferrari, Fantechi and Gnesi, 2012; Ferrari et al., 2013; Huhn and Milius, 2014; Basile et al., 2018; ter Beek et al., 2019), can be connected to a study using UTAUT. While all of these factors can be of great importance and all worthy of including in a study using UTAUT, maybe some of them should be initially prioritized.
According to the authors' perspective, assessment of the definition of a learning curve can be more straightforwardly integrated into UTAUT. The learning curve can be associated with the perceived ease of use, and consequently, effort expectancy that is already a variable in UTAUT and is already used in studies. Available technical support and documentation can also be part of defining the learning curve for FMs tools as a variable in the study and can be subject to examination. Compatibility can be a second construct to be considered for the study of FMs with UTAUT and can be considered to be a construct to support the variable of facilitating conditions. Compatibility, as well, is one of the notions that has been used in UTAUT before; however, in a study of FMs adoption, it can have more of technical meaning, rather than a notion that is connected to an individual or organizational beliefs or needs, as originally considered in UTAUT (Tan, 2013).

\section{Conclusion}

The current situation regarding FMs and their adoption to railway signaling applications can be considered to be in a stage where FMs are not utilized to their full potential. While there are enough tools and some successful applications, the understanding of the diffusion of their adoption remains a trivial task. Besides, from previous studies, it is noticeable that the use of a standard toolkit has not arisen yet, and different organizations use different approaches according to each team's or engineer's perspective and background. While there is not, however, a systematic longitudinal study or approach to the topic of adoption, several research questions arise (Rizopoulos et al., 2020), and strategical approaches on how to integrate FMs into 
current processes and projects will be hard to be developed.

Moving forward and regarding the future, FMs can potentially be one of the supporting scientific disciplines that will help achieve a unified European railway signaling network. It is expected that the number of FMs applications will rise and that they will also vary to the level of abstraction of their application regarding the knowledge that is required to apply the tools. Some high-level tools will be necessary for most of the projects on a system level, but also lowlevel tools will be essential to guarantee the formal development and formal verification of properties of subsystems. The study of the adoption of FMs can benefit significantly from this since more industrial data will be available on metrics that were monitored during projects, and by careful comparison, further evidence can be produced. In that manner, studies of the adoption of FMs will not have to solely depend on empirical evidence that is acquired through questionnaires or interviews.

The analysis presented in this article is another steppingstone in the broader view on the systematic study of the adoption of innovative technologies in railways and railway signaling. According to the authors' perspective, there is an immense need for adoption studies, as FMs are only one of the potential scientific methods and emerging technologies (based on the scientific methods) that will be needed to be adopted towards the modernization of railway networks. By presenting and analyzing the FMs use cases that can lead to potential demand outside the current needs that stem from legislation, it is aimed that further directions and insights are shared about future demand estimation for FMs. All of the work in the adoption of FMs should lead to the creation of a business case for FMs in railway signaling that can actually present quantitative data on the benefits of FMs in railway signaling projects.

\section{Acknowledgements}

This work has been supported by the Swedish Trafikverket as a part of the Shift2Rail research programme.

\section{References}

Ajzen, I. 1985. From Intentions to Actions: A Theory of Planned Behavior. Action Control: From Cognition to Behavior, 11-39.

Bacherini, S. et al. 2006. A Story About Formal Methods Adoption by a Railway Signaling Manufacturer. In Lecture Notes in Computer Science (including subseries Lecture Notes in Artificial Intelligence and Lecture Notes in Bioinformatics), 179-189.

Baregheh, A.; Rowley, J.; Sambrook, S. 2009. Towards a multidisciplinary definition of innovation, Management Decision 47(8): 1323-1339.

Basile, D. et al. 2018. On the Industrial Uptake of Formal Methods in the Railway Domain, In Lecture Notes in Computer Science (including subseries Lecture Notes in Artificial Intelligence and Lecture Notes in Bioinformatics). 20-29.

Batty, M. et al. 2012. Smart cities of the future, The European Physical Journal Special Topics 214(1): 481-518.

ter Beek, M. H. et al. 2019. Adopting Formal Methods in an Industrial Setting: The Railways Case. In International Symposium on Formals Methods, 762-772.

Behm, P. et al. 1999. Météor: A successful application of B in a large project. In Lecture Notes in Computer Science (including subseries Lecture Notes in Artificial Intelligence and Lecture Notes in Bioinformatics), 369-387. 
Bowen, J. P.; Hinchey, M. G. 1995. Seven More Myths of Formal Methods, IEEE Software 12(4): 34-41.

Christou, C.; Eliophotou-Menon, M.; Philippou, G. 2004. Teachers' concerns regarding the adoption of a new mathematics curriculum: An application of CBAM, Educational Studies in Mathematics 57(2): 157-176.

Cimatti, A. et al. 1998. Formal Verification of a Railway Interlocking System using Model Checking, Formal Aspects of Computing 10(4): 361-380.

Coccia, M. 2009. Measuring the impact of sustainable technological innovation, International Journal of Technology Intelligence and Planning 5(3): 276-288.

Crossan, M. M.; Apaydin, M. 2010. A Multi-Dimensional Framework of Organizational Innovation: A Systematic Review of the Literature, Journal of Management Studies 47(6): 1154-1191.

Davis, F. D. 1989. Perceived Usefulness, Perceived Ease of Use, and User Acceptance of Information Technology, MIS Quarterly 13(3): 319-340.

Douthwaite, B.; Keatinge, J. D. H.; Park, J. R. 2001. Why promising technologies fail: the neglected role of user innovation during adoption, Research Policy 30(5): 819-836.

Edison, H.; bin Ali, N.; Torkar, R. 2013. Towards innovation measurement in the software industry, Journal of Systems and Software 86(5): 1390-1407.

Essamé, D.; Dollé, D. 2006. B in large-scale projects: The canarsie line CBTC experience. In Lecture Notes in Computer Science (including subseries Lecture Notes in Artificial Intelligence and Lecture Notes in Bioinformatics), 252-254.

Fantechi, A.; Flammini, F.; Gnesi, S. 2014. Formal methods for railway control systems, International Journal on Software Tools for Technology Transfer 16(6): 643-646.
Ferrari, A. et al. 2013. Model-Based development and formal methods in the railway industry, IEEE Software $30(3): 28-34$.

Ferrari, A.; Fantechi, A.; Gnesi, S. 2012. Lessons Learnt from the Adoption of Formal Model-Based Development. In Lecture Notes in Computer Science (including subseries Lecture Notes in Artificial Intelligence and Lecture Notes in Bioinformatics), 24-38.

Fuller, F. F. 1969. Concerns of Teachers: A Developmental Conceptualization, American Educational Research Journal 6(2): 207-226.

Hall, G.; Wallace, R.; Dossett, W. 1973. A developmental conceptualization of the adoption process within educational institutions, Research and Development Center for Teacher Education, The University of Texas, Austin. USA. 44 p.

Goverde, R. M. P.; Corman, F.; D’Ariano, A. 2013. Railway line capacity consumption of different railway signalling systems under scheduled and disturbed conditions, Journal of Rail Transport Planning \& Management 3(3): 78-94.

Gruner, S. et al. 2013. Towards a Formal Methods Body of Knowledge for Railway Control and Safety Systems, FM-RAIL-BOK Workshop 2013. Technical University of Denmark. DTU Compute-Technical Report-2013 No. 20.

Gruner, S.; Kumar, A.; Maibaum, T. 2016. Towards a body of knowledge in formal methods for the railway domain: Identification of settled knowledge. In Communications in Computer and Information Science, 87-102.

Hall, A. 1990. Seven myths of formal methods, IEEE Software 7(5): 11-19.

Hall, G. E. 1979. The Concerns-Based Approach to Facilitating Change, Educational Horizons 57(4): 202208. 
Hauschildt, J. et al. 2016. Innovationsmanagement. Vahlen, Munich, Germany.

Haxthausen, A. E. 2010. An introduction to formal methods for the development of safety-critical applications. DTU Informatics Technical University of Denmark. Denmark.

Huhn, M.; Milius, S. 2014. Observations on formal safety analysis in practice, Science of Computer Programming. 80(Part A): 150-168.

Iacono, M. P. et al. 2012. Knowledge creation and inter-organizational relationships: The development of innovation in the railway industry, Journal of Knowledge Management 16(4): 604-616.

Indrawati; Yusliansyah, S. 2017. Adoption factors of online-web railway ticket reservation service (A case from Indonesia). In 2017 5th International Conference on Information and Communication Technology, ICoIC7 2017, 1-6.

Karg, S. et al. 2016. Model-driven software engineering in the openETCS project. In Proceedings of the ACM/IEEE 19th International Conference on Model Driven Engineering Languages and Systems - MODELS '16. 238-248.

Kline, S. J. 1985. Innovation Is Not a Linear Process, Research Management 28(4): 36-45.

Laurent, O. 2010. Using Formal Methods and Testability Concepts in the Avionics Systems Validation and Verification (V\&V) Process. In 2010 Third International Conference on Software Testing, Verification and Validation, IEEE. 1-10.

Madigan, R. et al. 2016. Acceptance of Automated Road Transport Systems (ARTS): An Adaptation of the UTAUT Model. In Transportation Research Procedia, 14: 2217-2226.
Madigan, R. et al. 2017. What influences the decision to use automated public transport? Using UTAUT to understand public acceptance of automated road transport systems, Transportation Research Part F: Traffic Psychology and Behaviour 50: 55-64.

Marchewka, J.; Liu, C.; Kostiwa, K. 2007. An Application of the UTAUT Model for Understanding Student Perceptions Using Course Management Software, Communications of the IIMA 7(2): Article 10.

O’Hara, M. T.; Watson, R. T.; Kavan, C. B. 1999. Managing the three Levels of Change, Information Systems Management 16(3): 63-70.

Palmqvist, C. W.; Olsson, N. O. E.; Hiselius, L. W. 2017. Some influencing factors for passenger train punctuality in Sweden, International Journal of Prognostics and Health Management, 8(7): 1-13.

Puthur, J. K.; George, A. P.; Mahadevan, L. 2020. Understanding citizen's continuance intention to use e-government services: the case of the Indian railway e-ticket booking site, International Journal of Business Information Systems 34(2): 183-203.

Ren, M. 2019. Why technology adoption succeeds or fails: an exploration from the perspective of intraorganizational legitimacy, The Journal of Chinese Sociology 6(1): 1-26.

Rizopoulos, D. et al. 2020. Research Directions Regarding the Adoption of Formal Methods in the Railway Signaling sector: Determinants and next steps for future-proof railways. In Computers in Railways XVII, $75-86$.

Rogers, E. M. 2003. Diffusion of Innovations. Free Press, New York, USA, 551 p. 
Smith, R. A. 2003. Railways: How they may contribute to a sustainable future. In Proceedings of the Institution of Mechanical Engineers, Part F: Journal of Rail and Rapid Transit 217(4): 243-248.

Foon, Y. S.; Fah B. C. Y. 2011. Internet Banking Adoption in Kuala Lumpur: An Application of UTAUT Model, International Journal of Business and Management 6(4): 161-167.

Souyris, J. et al. 2009. Formal verification of avionics software products. In Lecture Notes in Computer Science (including subseries Lecture Notes in Artificial Intelligence and Lecture Notes in Bioinformatics), 532-546.

Straub, E. T. 2009. Understanding technology adoption: Theory and future directions for informal learning, Review of Educational Research 79(2): 625-649.

Tan, P. J. B. 2013. Applying the UTAUT to Understand Factors Affecting the Use of English E-Learning Websites in Taiwan, SAGE Open 3(4): 1-12.

Venkatesh et al. 2003. User Acceptance of Information Technology: Toward a Unified View, MIS Quarterly 27(3): 425-478.

Venkatesh et al. 2012. Consumer Acceptance and Use of Information Technology: Extending the Unified Theory of Acceptance and Use of Technology, MIS Quarterly 36(1): 157-178.
Venkatesh, V. 2000. Determinants of Perceived Ease of Use: Integrating Control, Intrinsic Motivation, and Emotion into the Technology Acceptance Model, Information Systems Research 11(4): 342-365.

Venkatesh, V.; Bala, H. 2008. Technology Acceptance Model 3 and a Research Agenda on Interventions, Decision Sciences 39(2): 273-315.

Venkatesh, V.; Davis, F. D. 2000. A Theoretical Extension of the Technology Acceptance Model: Four Longitudinal Field Studies, Management Science 46(2): 186-204.

Vu, L. H. 2015. Formal Development and Verification of Railway Control Systems - In the context of ERTMS/ETCS Level 2. Technical University of Denmark. DTU Compute PHD-2015 No.395.

Vu, L. H.; Haxthausen, A. E.; Peleska, J. 2014. A Domain-specific language for railway interlocking systems. In FORMS/FORMAT 2014 - 10th Symposium on Formal Methods for Automation and Safety in Railway and Automotive Systems, 200-209.

Woodcock, J. et al. 2009. Formal methods, ACM Computing Surveys 41(4): 1-36.

Ye, J.; Zheng, J.; Yi, F. 2020. A study on users' willingness to accept mobility as a service based on UTAUT model, Technological Forecasting and Social Change 157: 120066. 